\title{
The Construction of Global 3D Scene Platform for Combat Oriented and Multi Task Planning
}

\author{
Daowei Liu" a , Jie Xiong ${ }^{1, b}$, Yijia Pan ${ }^{1, c}$ and Liang Liang ${ }^{1, c}$ \\ ${ }^{1}$ Luoyang Electronic Equipment Test Center of China, Luoyang, China \\ a66359150@qq.com, b1650605@qq.com, '942908@qq.com,'9429208@qq.com
}

Keywords: Combat scenario; Mission planning; Global 3D scene; Cooperative combat

\begin{abstract}
This paper focuses on the global three-dimensional scene construction technology, combining the 2D map and combat formation scenario technology, combined with 2D and 3D scene of mission planning technology and operational deployment of data driven interface design technology and so on. The system in the practical application, improve the operational scenario and mission planning system and combat simulation model portability and reusability; integration of existing data driven and 3D simulation platform, completed the system coordination policy, support the development of collaborative technology of intelligent system.
\end{abstract}

\section{Introduction}

Combat simulation system of virtual scene with virtual reality technology has been rapid development, the combat system is difficult to meet the local virtual scene simulation of modern warfare multi-level coordination requirements, and so the combat simulation system of virtual scene must build a global terrestrial environment, marine environment, air environment and space environment. Through the study of the global three-dimensional scene construction technology, combining the 2D map and combat formation scenario technology, combined with 2D and 3D scene of mission planning technology and operational deployment of data driven interface design method to realize the oriented scenario and multi task programming global 3D scene platform, on the battlefield in the environment of both red and blue for attack and defense, combat scenario and multi task planning. Can improve the combat scenario and combat simulation model portability and reusability; repeated development of combat system so as to save a lot of resources effectively; the integration of the existing data driven and 3D simulation platform, complete system coordination policy, support the development of the intelligent system of collaborative technology[1,2].

\section{Global 3D Scene Construction Technology}

It is the basis of the combat simulation for the global 3D battle scene generation and the platform construction. It provides the data security and the visual scene of the situation observation for the combat scenario generation system and the multi task planning system. The global three-dimensional scene mainly includes the sea, the land, the sky and the space and so on, including the massive terrain and the image data, the massive multi-dimensional information and the special effect. How to integrate the global massive terrain image raster data and vector data such as the border line, provincial boundary line, landmark and so on? How to accurately load DSM (Digital Surface Model, digital surface model) and surface features in the global coordinate system? How to make the scene more realistic, special effects, natural scenes closer to reality? Can you make the scene more diverse, can not be simulated on the basis of the global three-dimensional simulation of infrared scenes to support combat simulation? For the above problems, this paper 
presented the battle to global 3D scene platform and multi task programming, is divided into three sub modules: Global combat environment platform, mission planning platform, programming and operational scenario generation platform. Mainly has the following functions: 1) the organization of 3D geographic terrain data and processing functions: effectively organizing and processing the original data in different files, different projection model, different range of data and different data accuracy, geographic scheduling and display, providing indirect high efficient and unified interface. The dynamic display of the data with different resolution is layered and displayed. 2)display natural weather multidimensional information: fast real information such as the natural environment, the deployment of troops, combat progress, weather conditions and electromagnetic environment together to provide a dynamic and complex battlefield battlefield commanders, visualization and analysis of global battlefield environment information acquisition. 3) the function of data driven interface: it can integrate the simulation data of the scenario and other combat system in the platform, and display the 3D data in the system. 4) browsing and user interaction observation function of 3D scene: Global battlefield environment visualization and analysis software Google Earth to take a similar way to manage the world from space, the different terrain labeling organization and scheduling on the earth, can enter the local area of the corresponding terrain roaming, observed the first viewpoint or third flexible view and is able to fly combat entities $[3,4]$.

\section{Research on Combat Scenario and Multi Task Planning Based on 2D Map}

Scenario is the battle of scripts, program, is a preview and prediction process of the actual battlefield combat, combat commanders and combat strategy intention through certain forms, according to the observations of global three-dimensional scene construction technology to carry on the battlefield situation and the trend of deduction. In the battlefield environment is becoming increasingly complex, task deployment scenario become increasingly diverse circumstances, individual entities are usually hard to meet the needs of target penetration, need more bombs or multi operational entities to complete the established cooperative combat intention and scenario situation, multi task programming is to achieve the overall operational efficiency and cost optimization the minimum consumption to determine the operation from the target area to combat tasks throughout the flight time, in accordance with the timing control plan of missile or aircraft fleet combat mission group[5].

Combat Deployment. The design of combat simulation is a complicated and gradual process, which involves a lot of combat elements, such as the basic information of combat, combat environment information, operational information and so on. How to convert the data files from the existing simulation system into the format that our system can support. There are a number of different entities in the system, and there are different properties, various entities such as: Property launch platform launch platform launch platform type, position, course and speed of the launch platform launch platform, with the missile launch platform, missile launch platform, information stations, distribution stations transmit radius; missile type the missile, missile flight time, the properties of missile flight status, missile real-time position, missile flight speed, pitch angle, yaw angle of missile missile, missile roll angle etc.. In this paper mainly adopts the 3D database to store operational organization of each operational entities, in the study of weapon attributes description, weapons, weapons physics simulation model of behavior model, the $3 \mathrm{D}$ construction of database table to store[6]. To store the data by constructing a three-dimensional combat database, on the basis of generating the deployment of combat entities toolbox for militaryforce, with the help of scene management tree to manage the scene of the combat entity, the final entity in the global remote sensing map or global 3D scene model to generate accurate positioning dynamic combat 
deployment and combat scenario. The combat entity is to combat the most basic elements of the set, but also in the combat zone, subject to the simulation of essential elements, through the deployment of military region can result to exercise restraint and influence the situation even combat simulation. In the system, there are no fly zone, gathering area, target area, patrol area, standby area and search area. The format of the regional data package defined in this paper is shown in table 1.

Table 1 the definition of region description data packet

\begin{tabular}{|l|l|l|l|}
\hline Order number & Data name & Data type & Data identification \\
\hline 1 & Packet identifier & unsigned short & pkgID \\
\hline 2 & Packet length & unsigned short & pkgLen \\
\hline 3 & Packet update count & unsigned short & pkgCount \\
\hline 4 & A region identifier or not & unsigned short & IsUsable \\
\hline 5 & Area data block identifier & unsigned short & RegionID \\
\hline 6 & Regional data block area & pkgRegion2 & Region \\
\hline 7 & Block data attribute 1 & unsigned short & Property1 \\
\hline 8 & Block data attribute 2 & float & Property2 \\
\hline 9 & Block data attribute 3 & pkgtime & Property3 \\
\hline
\end{tabular}

The same can be stored in similar organization method of operational entities based on the definition of regional data packets, and definitions in the database area data table, add the icon in the area of the combat entity in the toolbox, in the scenario of the deployment area, you can drag the region to the designated location, through setting up the regional data block regional and regional data block properties, mainly types of regional scope, shape and area. Operational deployment is shown in Fig. 1.

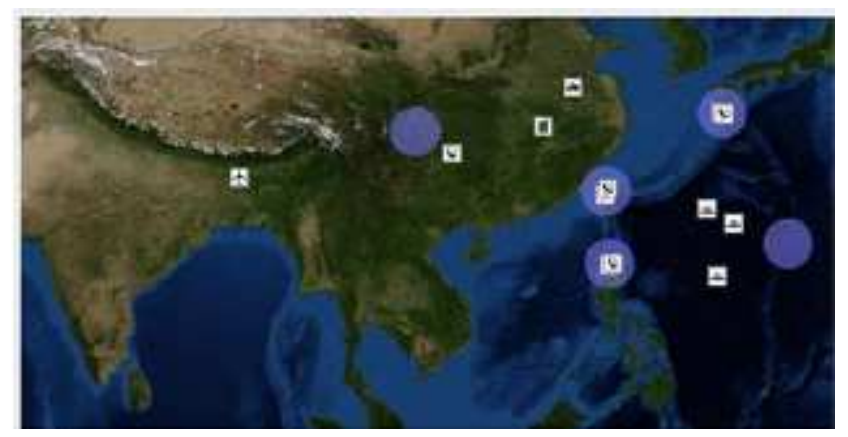

Figuer 1. Combat entities and regional deployment

In the use of battle scenario generated scenario files to record the simulation scenario data deployment toolbox in this system to file mainly contains the following information: the scenario of basic information, including basic properties including scenario logo, name, purpose, scenario creation time, the warring parties composition, operational time, combat, combat intention etc.. The combat environment information, including the natural weather environment, the battlefield geographical environment and other factors. Combat information, including identification, force type, location, property of the enemy and the enemy, the command relationship, weapon identification, name, type, location, membership. Combat mission information, including task identification, type, start time, end time, combat areas, combat forces and weapons deployment, operational objectives, etc.. So in the system to generate the.Scene file format XML file, the following figure is the interception of the system to determine the file, as shown in Fig.2. 


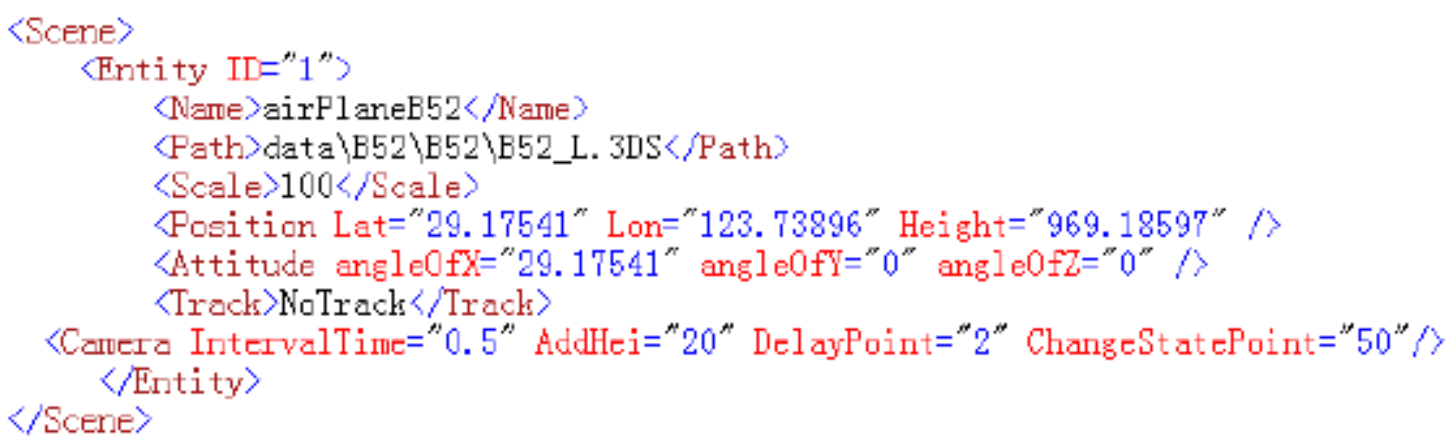

Figure 2. File format diagram

In the scenario data file, if you want to interact with words such as HLA/DWK system interactive simulation system of the other, is mainly based on the design of the data interface, sends the HLA/DWK combat deployment of federate over the data using data interface to parse and generate the corresponding. Scene file, the system can be in combat to deploy and combat drills. Combat deployment effect as shown in Fig. 3.
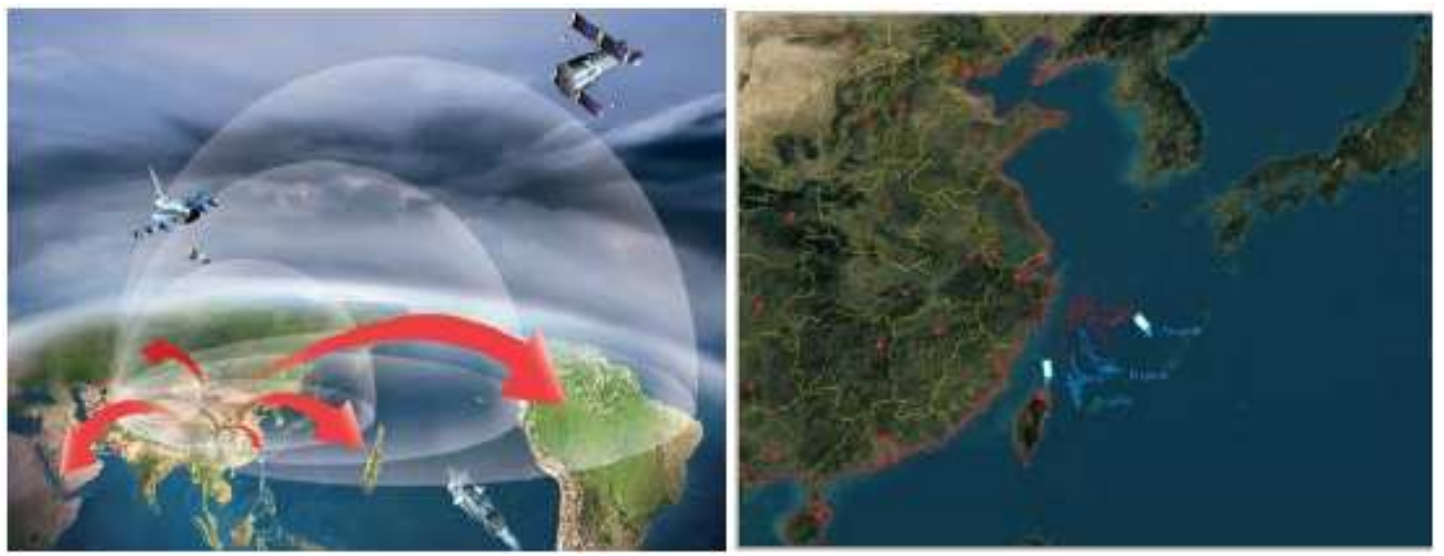

Figure 3. Combat deployment in three-dimensional and two-dimensional renderings

Multi Task Planning. With the operation of multi task planning module in this system to interact through the module to the XML file, the file to the scenario task is determined, combined with the scenario of environmental information, consider battlefield deployment areas and to detect enemy defense scope constraints. A multi task programming algorithm (dynamic programming, genetic algorithm, Voronoi chart, $\mathrm{A}^{*}$ algorithm) to find out the optimal flight path and meet the scenario, the multi task planning results given the corresponding collaborative aircraft or missile group. Multi task planning is actually a constraint analysis and task planning scheme, due to many factors and environmental conventions are too complex, combined with the actual system, can simplify the implementation process, the main consideration of some key variables, by setting the control point and simple constraint through curve fitting to create a smooth track network. Of course, this system can also be complex presentation and battlefield multi task programming inference, mainly through the professional mission planning system of the transmitted data to simulate the flight task, see the specific design of shared memory data interface.

\section{Design and Research of Data Driven Interface}

Data Driven Interface. Including the global 3D scene and combat mission planning platform 
provides a data driven interface, based on HLA (High Level Architecture), DWK (Digital Weapon Kit), shared memory multiple ways, with interoperability, flexibility, encapsulation, reuse, better. You can customize any of the above interface or multiple interfaces according to the actual needs of users, the multiple simulation system of space distribution through the public communication network together, in the simulation time and simulation environment unified to complete complex scenario and multi task planning simulation tasks, collaborative combat simulation tasks, repeated development the development of combat system so as to save a lot of resources to avoid. It can significantly improve the efficiency of the combat scenario generation, shorten the system development cycle, complete the coordinated operation policy of the system, and support the development of intelligent system coordination technology. The system realizes the interface with the HLA/DWK architecture: 1) the scene model driven support HLA distributed simulation, support HLA federate data transceiver and interaction. 2, through the scene simulation data interpolation extrapolation method to achieve smooth flow of the scene. The realization of the system and the shared memory interface process: 1) against the scene model driven support for shared memory simulation model. 2) get the data packet through the network and analyze the data. 3) deal with the data, the battle will be deployed in the global three-dimensional battlefield environment.

HLA/DWK interface design. HLA provides three layers of application: combat simulation application layer, simulation operation management layer and the underlying network communication management layer. Each layer can be developed independently, with flexibility, diversity and scalability. DWK provides various simulation tools and modules, before simulation in DWK and Scenarior (Modellor modeling module) (director control module), SDK software development kit, Tester (test module) to support the development and production system. In this paper, we use the Scenarior control module DWK and the 3D rendering module in this system to realize the data transmission and interaction between the heterogeneous simulation system. Scenarior is the main operation scenario simulation function and management control, can carry out professional scenario deployment and combat force, management and control simulation type, evaluates the efficiency or tactical training, single trip or simulation many times simulation, the whole simulation and selection of each trip at the end of the simulation conditions, federal members of the operation the deployment, display the simulation clock, wall clock, simulation and adjustment of the beat, pause, stop, run the simulation, display provides a global two-dimensional situation, realize the trend of navigation, Scenarior director control effect in Fig. 4.

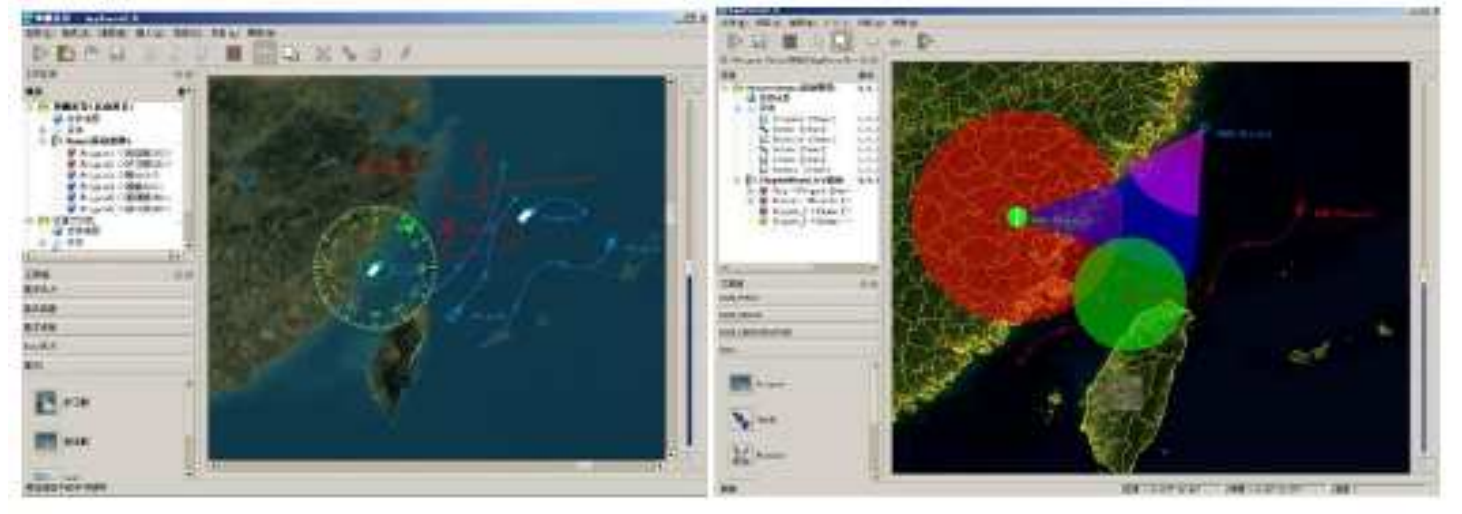

Figure 4. Director control effect diagram

The data interface based on DWK is to receive the data from the Scenarior module to our system and analyze and render it. The specific process is as follows: 1) the system as a three-dimensional display of federal members to join the Federation of DWK. 2) according to the state and property of 
the federate members of each combat entity in the federal director control module, the data class is designed to encapsulate the entity attributes and states. 3 , the system has a thread to read the data packet, and according to the information required by the system, the packet analysis, remove the effective packet information. 4, the effective data is sent to the callback class in the system, and each frame is updated in the system.

Shared Memory Interface Design. Between the system and the intelligent anti-ship system interactive through shared memory interface, there are a lot of communication between processes, mainly in shared memory, named pipes and anonymous pipes, transmit messages, sharing is more common among processes using the same physical memory space memory, it is based on the same physical the memory mapped to the virtual space to realize the process of different. Due to the mapping to different processes in the virtual space, different processes can be used directly, do not need to copy the memory, so shared memory is more efficient than other mechanisms. It provides a mechanism for efficient two-way communication between any number of processes, which can obtain high performance with low cost, and is the best solution for fast data exchange. First initialize the shared memory framework, the distributed intelligent anti-ship system simulation of battlefield and combat scenario, through the TCP/IP protocol to send combat data encapsulated packets into the shared memory area, the polling thread in the system constantly sharing area, every 20ms number according to PKG Data Update data structure to judge if the data if the data update will be on the P Share Infor- $>$ m_data Update in the data analysis system, remove the required data, and update the rendering visualization battle scene in the callback trend.

\section{Conclusion}

This paper focuses on the global three-dimensional scene construction technology, combining the 2D map and combat formation scenario technology, combined with 2D and 3D scene of mission planning technology and operational deployment of data driven interface design technology and so on, to achieve a global three-dimensional scene scenario and multi task programming platform. But some problems still exist between the actual application in the theoretical study, global three-dimensional scene visualization, weapon model, terrain and images in the import system after the loss of precision, lack of interaction in the flight combat entity physics engine support lack of sense of reality. The two is the study of the timing control mechanism of the entity cooperation in the multi task planning, the research and application of the more complex and more scientific task planning algorithm.

\section{References}

[1] P.Hu. Design and implementation of visual operation scenario generation system based on Web services [M]. Zhengzhou Information Engineering University, 2010.

[2] X.Q.Hu. Virtual reality technology [M]Beijing: Beijing University of Posts and Telecommunications press,2005: 30-50.

[3] Jiyeong Lee. A Spatial Access-Oriented Implementation of a 3-D GIS Topological Data Model for Urban Entities [J]. GeoInformatica, 2004, 8(3).

[4] Kaab A. Combination of SRTM3 and Repeat ASTER Data for Deriving Alpine Glacier Flowv elocities in The Bhutan Himalaya[J].Remote Sensing of Environment, 2005, (94):463-671.

[5] Oky D A P,Ayako E,Ryuzo Yo,et al. Supervised land form classification of North-east Honshu from DEM-derivedthematic maps[J] .Geomorphology, 2006, 78:373-386.

[6] Jeff Abbott. Military Scenario Definition Language Overview[Z]. OneSAF MSDL Task Lead. Orlando USA: the Simulation Interoperability Standards Organization, 2005. 
[7] The design of level maintenance equipment warehouse based on HLA/RTI[J].Ordnance Industry Automation, 2006,(06).. 